\title{
Evaluation of Itch by Using NC/NgaTnd Mice: A Model of Human Atopic Dermatitis
}

\author{
Akane Tanaka and Hiroshi Matsuda \\ Laboratory of Veterinary Molecular Pathology and Therapeutics, Division of Animal Life Science, Graduate School, \\ Institute of Agriculture, Tokyo University of Agriculture and Technology, 3-5-8 Saiwai-cho, Fuchu, Tokyo 183-8509, Japan \\ Correspondence should be addressed to Hiroshi Matsuda, hiro@cc.tuat.ac.jp
}

Received 15 September 2010; Accepted 28 October 2010

Academic Editor: Andrea Vecchione

Copyright ( $\odot 2011$ A. Tanaka and H. Matsuda. This is an open access article distributed under the Creative Commons Attribution License, which permits unrestricted use, distribution, and reproduction in any medium, provided the original work is properly cited.

\begin{abstract}
Atopic dermatitis $(\mathrm{AD})$ is the extremely complicated syndrome that various abnormalities develop in a heap. There are various factors in patients for the onset and exacerbation of $\mathrm{AD}$, including genetic cofactors of individuals, environmental factors, the failure of the skin barrier function, unfavorable regulation of the immune system, and the hypersensitivity of sensory nerves. In recent years, there have been many trials of the drug discovery that targets itch, because itch is one of the most serious clinical symptoms of $\mathrm{AD}$. The selection of the suitable animal model that represents the condition of patients, as well as innovative analyzing protocols that can precisely evaluate itch, is indispensable for investigation of an effective drug for AD. In the paper, the unique spontaneous animal model for $\mathrm{AD}(\mathrm{NC} / \mathrm{NgaTnd}$ mice) and the novel quantification system of the laboratory animals that may bring a great progress in the future study of itch are outlined.
\end{abstract}

\section{Introduction}

Atopic dermatitis $(\mathrm{AD})$ is a chronic and relapsing skin disorder with allergic immune responses resulting in the hyperproduction of $\operatorname{IgE}[1]$. The most of the patients have genetic cofactors promoting manifestation and/or family history of allergic disorders, including anamnesis either or some of bronchial asthma, allergic rhinitis, conjunctivitis. Increased numbers of activated circulating $\mathrm{CD} 4^{+}$and $\mathrm{CD} 8^{+}$ $\mathrm{T}$ cells and marked infiltration of $\mathrm{CD} 4^{+} \mathrm{T}$ cells into the dermis are characteristic features of patients with AD. In particular, $\mathrm{T}$ cells bearing the cutaneous lymphocyte antigen with features of allergen-specific $\mathrm{T}$ cells of the Th2-type are recruited. The initial phase of $\mathrm{AD}$ is dominated by Th2 type T cells producing IL-4, IL-5, and IL-13; at the subsequent chronic phase, the number of Th1 cells producing IFN- $\gamma$ increases. Not only $\mathrm{T}$ cells but also keratinocytes are capable of aggravating the inflammatory reaction in the skin through upregulated release of some cytokines and chemokines. Furthermore, barrier dysfunction of atopic skins is likely to play a pivotal role in the exacerbation of clinical conditions [2]. Contact or invasion of environmental antigens such as house dust mites exacerbates clinical symptoms. Itch is one of the most serious clinical symptoms of $\mathrm{AD}$, and repeated itchingscratching episodes may be greatly involved in acceleration and severity of dermatitis $[1,3,4]$. Epidermal hyperplasia, as well as infiltration of mononuclear cells and eosinophils, and degranulation of mast cells at the affected skins, is the most typical pathological manifestations of $\mathrm{AD}$ [3]. Since the background is multifactorial and the underlying predispositions is involved, the pathogenesis of $\mathrm{AD}$ is quite complex. For understanding pathogenic pathways of $\mathrm{AD}$, suitable animal models are necessary, which may lead to the development of new drugs with great benefit for patients with $\mathrm{AD}[5,6]$.

\section{Spontaneous Model for Human AD: NC/NgaTnd Mice}

Microscopic features of skins obtained from NC/NgaTnd mice raised in SPF (a) or conventional (b) circumstances.

NC/NgaTnd mice, an inbred strain originated from Japanese fancy mice, spontaneously develop itchy dermatitis 


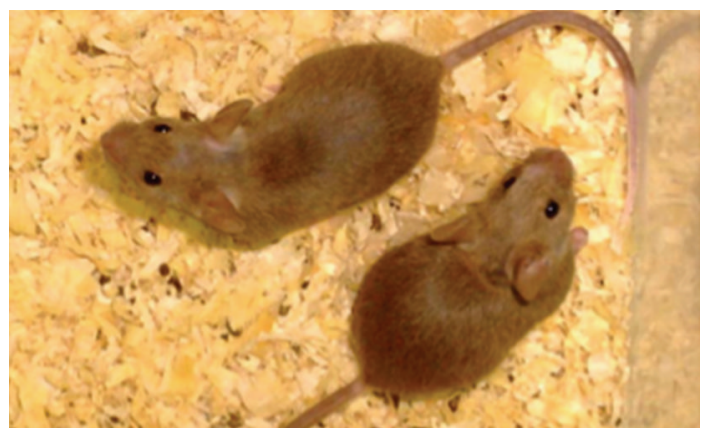

(a)

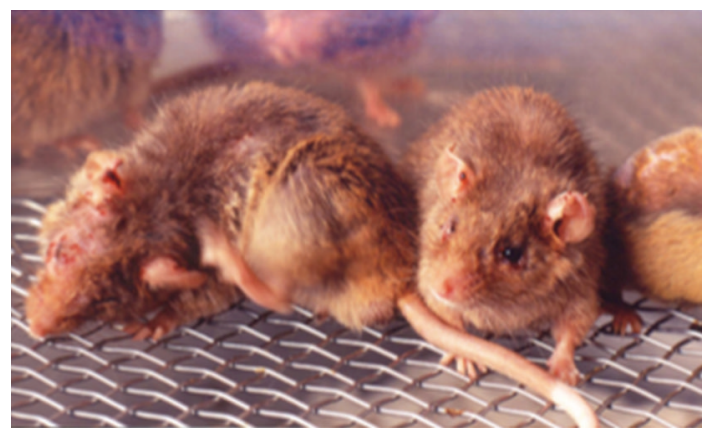

(b)

Figure 1: NC/NgaTnd mice maintained in air-regulated SPF (a) or air-unregulated conventional circumstances (b).

in an air-unregulated conventional circumstance from 6 to 8 weeks of age (Figure 1). On the other hand, skin lesions do not develop when they are raised in air-regulated specific pathogen-free (SPF) conditions (Figure 1). We have analyzed aspects manifested in $\mathrm{NC} / \mathrm{NgaTnd}$ mice immunologically, pathologically, dermatologically, and molecular biologically and found that clinical symptoms in NC/NgaTnd mice are quite similar to those found in human $\mathrm{AD}$ [7-9]. Thereafter, as the first spontaneous $\mathrm{AD}$ model mouse, $\mathrm{NC} / \mathrm{NgaTn}$ mice attract attention from researchers of the world. NC/NgaTnd mice are estimated as an appropriate animal model for the screening of novel therapeutic agents for $\mathrm{AD}$. In affected skins of NC/Nga mice, epidermal hyperplasia, increase and degranulation of mast cells and recruitment of inflammatory cells are remarkable (Figure 2). In addition, IL-4 is produced in $\mathrm{CD}^{+}{ }^{+} \mathrm{T}$ cells and mast cells, and Th2-specific chemokines are overproduced [10], indicating that Th2type immune responses are upregulated. Exacerbation of dermatitis relates to the increase of total IgE levels and eosinophil numbers in circulation. Contents of ceramide in skins are decreased in NC/NgaTnd mice before dermatitis becomes remarkable, and it became clear that transepidermal water loss of affected skins was promoted as a result [11]. The Itch-scratch cycle contributes to the development of $\mathrm{AD}$ as a cofactor. Decreased production of semaphorin $3 \mathrm{~A}$ that has an activity to inhibit nerve growth factor-induced sprouting of sensory neurons is recently reported in the skin lesions of patients with $\mathrm{AD}$ and conventional $\mathrm{NC} / \mathrm{Nga}$ mice $[12,13]$, and repeated topical injection with recombinant

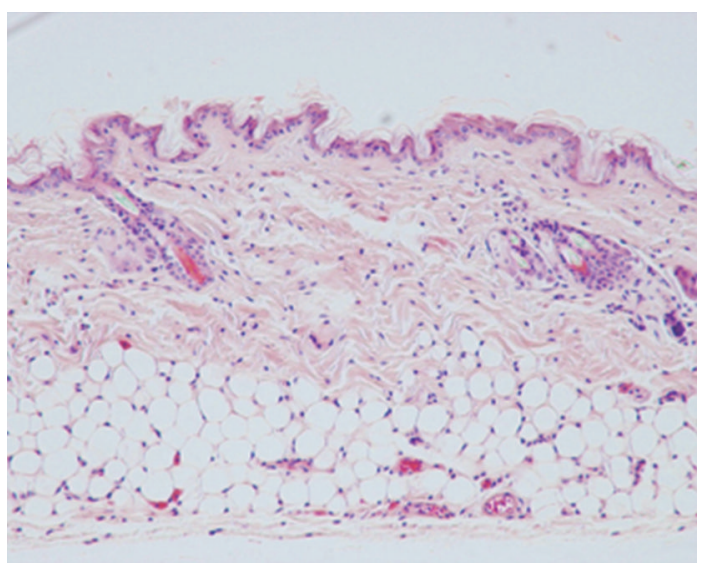

(a)

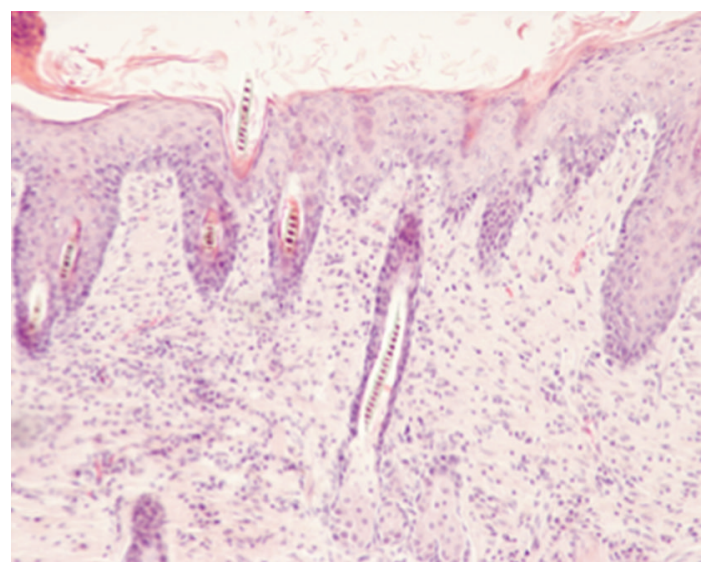

(b)

Figure 2: Generation of mast cell-deficient NC/NgaTnd mice. NC/NgaTnd mice with heterozygous (a) and homozygous (b) for the mast cell-deficient Wsh gene mutation.

semaphorin $3 \mathrm{~A}$ is effective to improve the skin lesions of conventional NC/Nga mice [13]. More recently, we have demonstrated that thymic stromal lymphopoietin released from keratinocytes of the skin lesions contributes to the early stage of $\mathrm{AD}$ in $\mathrm{NC} / \mathrm{NgaTn}$ (nice and peroxisome proliferators activated receptors gamma activation downregulates the onset of $\mathrm{AD}$ via inhibition of dendritic cell functions activated by thymic stromal lymphopoietin [14].

Researchers have to be careful because there are some sublines of the NC strain that rarely develops AD spontaneously. When experiments in the conventional circumstances are not applicable, allergic dermatitis can be induced by using repeated application protocols with haptens, including FITC or picryl chloride. However, the hapteninduced dermatitis is initiated by a Th1-mediated immune response. Recently, atopic dermatitis-like skin lesions can be reproduced by applying the extract of house dust mites to SFP NC/NgaTnd mice as an ointment. Furthermore, hairless $\mathrm{NC} / \mathrm{Nga}$ mice are generated by using the toxin receptormediated cell knockout (TRECK) method [15]. Hairless $\mathrm{NC} / \mathrm{NgaTn}$ mice allows us to apply therapeutic reagents 


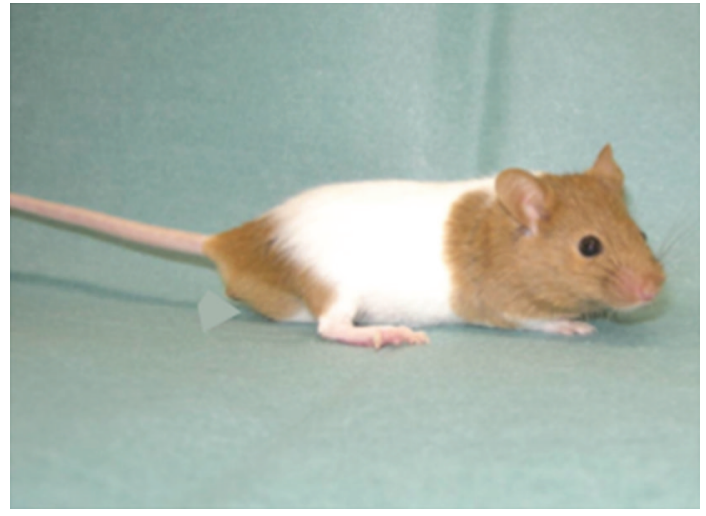

(a)

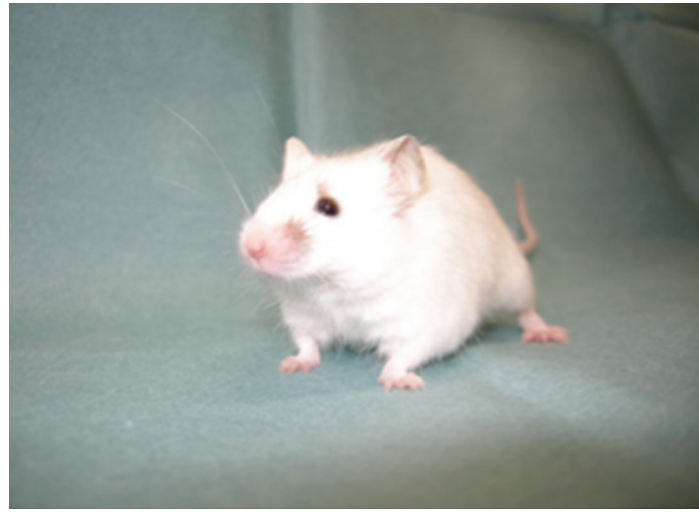

(b)

Figure 3: Microscopic features of skins obtained from NC/NgaTnd mice raised in SPF (a) or conventional (b) circumstances.

onto skins without shaving and to observe clinical symptoms quite easily.

In our laboratory, NC/NgaTnd mice have been backcrossed to specific gene-deficient mice such as matrix metalloprotease-9, Fc receptor $\gamma$ chain, protease-activated receptor 2 and succeeded in the construction of different congenic NC/NgaTnd mice deficient for these genes. Furthermore, mast cell-deficient NC/NgaTnd mice have been generated (Figure 3). Congenic gene manipulated $\mathrm{NC} /$ NgaTnd mice will provide more precise information concerning the involvement of certain genes in the development and/or exacerbation of AD.

\section{A Novel Scratch Analyzing System for Laboratory Mice: SCLABA-Real}

Reliable quantification of scratching behavior in laboratory mice is helpful for investigating itch mechanisms and for developing new drugs for allergic dermatitis. We identified a feasible parameter for quantification of scratching behavior in mice using a novel analyzing system, SCLABA-Real (Figure 4). Although intradermal injection with pruritogenic reagents into mice, precise measurement and evaluation of high-speed scratching behavior in mice have still been complicated. SCLABA-Real enabled us to analyze scratching behavior in real time without giving any kinds of stressful operations to a mouse $[16,17]$. The behavior of 4 mice is simultaneously recorded with a high-speed digital vision from the top of the exclusive cage placed on near-infrared light. A SCLABA-Real software accurately detects the algorithm that is specific for scratching behavior in mice and records data in real time. Effective antipruritic reagents can be selected by an accurate evaluation of scratching behavior in laboratory mice using a SCLABA-Real system.

The novel scratch analyzing system, SCLABA-Real, calculates "frame-to-frame difference" that is a mouse's migration area per frame by capturing animal behavior at the rate of 240 frames per second using the high-speed camera containing image processing system $[16,17]$. This whole new system allows us to quantify the number and duration of scratching behavior automatically by time-course analysis of "frame-to-frame difference" and evaluate the degree of itching from many directions.

The "frame-to-frame difference" analysis of the movement is the uniquely method realized by using the special camera in this system which can send images and the data to the hard disk of a computer instantly through the video server and LAN, which enable us to perform real-time and accurate analysis of scratching only. Prolonged measurement can be carried out because the stress on mice from the preliminary treatment and the environment is excluded. The recording platform has a built-in near-infrared light panel. Scratching behavior of mice can be recorded in a natural state using invisible near-infrared light. This system allows us to evaluate scratching behavior not only in the day time but also in the nighttime and darkness. The timer that activates the system automatically enables to start on analysis at night.

Although scratching behavior is the critical item for the drug development, its quantification by visual check has been quite subjective. However, this innovative product provides automatic analysis of scratching behavior in an objective and accurate manner.

\section{Remarks}

$\mathrm{AD}$ is a quite complicated syndrome with many abnormalities, including genetic, environmental, immunological, and dermatological cofactors. To select suitable animal models for each research is particularly important to obtain good outcomes. Therefore, researchers should know the characteristics, including advantages and disadvantages, of the model well. The spontaneous AD model, NC/NgaTnd mice have provided valuable information concerning the evaluation and selection of effective new drugs and supplements [18-21].

The drug development that targeted itch is prosperous, but the past trials for quantification of itch with a laboratory animal have been failed. SCLABA-Real is an apparatus of the extremely precise analyzing system for itch, that is totally different from conventional measuring systems. 


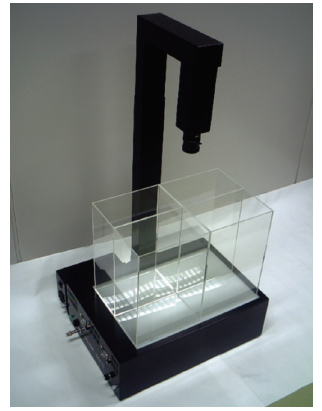

(a)

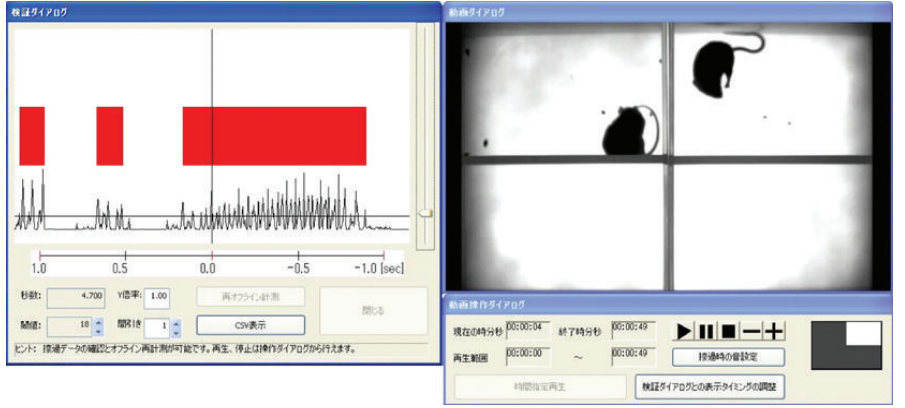

(b)

\begin{tabular}{|c|c|c|c|}
\hline & A & B & c \\
\hline 1 & No & StartFram & EndFrame \\
\hline 2 & 1 & 739 & 751 \\
\hline 3 & 2 & 1263. & 1287 \\
\hline 4 & 3 & 3138 & 3158 \\
\hline 5 & 4 & 5061 & 5107 \\
\hline 6 & 5 & 6903 & 6923 \\
\hline$\gamma$ & 6 & 7465 & 7492 \\
\hline 8 & 7 & 7540 & 7613 \\
\hline 9 & 8 & 7671 & 7704 \\
\hline 10 & 9 & 7744 & 7766 \\
\hline 11 & 10 & 7064 & 7940 \\
\hline 12 & 11 & 6519. & 8539 \\
\hline 13 & 12 & 19921 & 19539 \\
\hline 14 & 13 & 18442 & 19528 \\
\hline 15 & 14 & 19588 & 19713 \\
\hline 16 & 15 & 20009 & 20003 \\
\hline 17 & 16 & 26671 & 20598 \\
\hline 18 & 17 & 20741 & 20781 \\
\hline 19 & 18 & 20055 & 20914 \\
\hline 20 & 19 & 21218 & 21047 \\
\hline 21 & 20 & 24060 & 24112 \\
\hline 22 & 21 & 24160 & 24186 \\
\hline 23 & 22 & 27687 & 27726 \\
\hline 24 & 23 & 30860 & 30684 \\
\hline 25 & 24 & 32617 & 32640 \\
\hline 26 & 25 & 32660 & 32695 \\
\hline
\end{tabular}

(c)
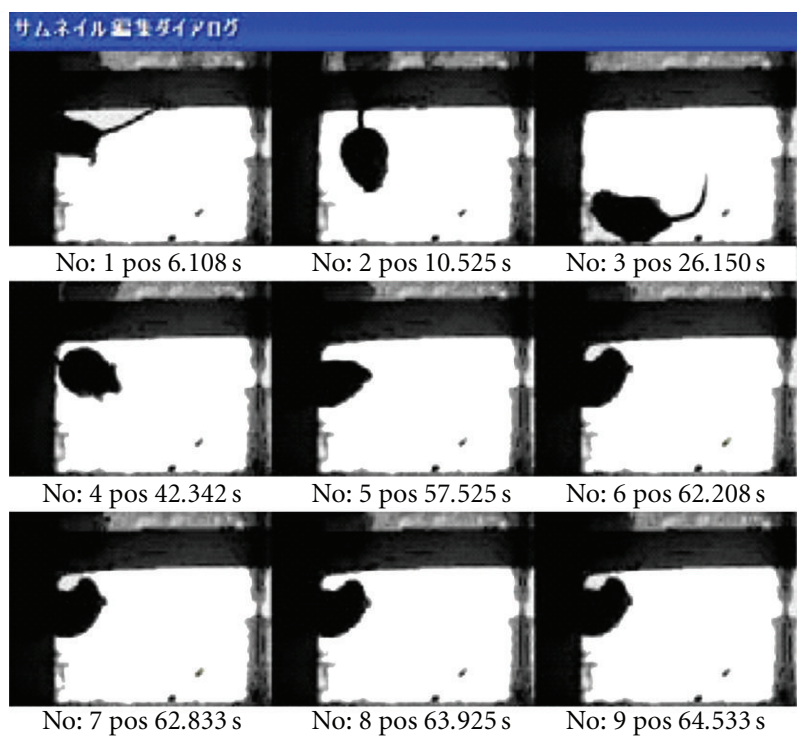

(d)

FIgURE 4: A novel scratch analyzing system, SCLABA-Real. (a) The high-speed camera containing image processing system and the recording platform with a built-in near-infrared light panel for real-time quantification of scratching behavior of mice. (b) Real time record and analyze window. (c) Analyzed data of scratching frequency and duration. (d) Validation window for each frame.

A spontaneous AD model NC/NgaTnd mice and a SCLABAReal system provide a possibility to develop the study of itch in the next generation.

\section{References}

[1] V. S. Beltrani, "Suggestions regarding a more appropriate understanding of atopic dermatitis," Current Opinion in Allergy and Clinical Immunology, vol. 5, no. 5, pp. 413-418, 2005.

[2] D. Y. M. Leung, M. Boguniewicz, M. D. Howell, I. Nomura, and Q. A. Hamid, "New insights into atopic dermatitis," Journal of Clinical Investigation, vol. 113, no. 5, pp. 651-657, 2004.
[3] E. Proksch, R. Fölster-Holst, and J. M. Jensen, "Skin barrier function, epidermal proliferation and differentiation in eczema," Journal of Dermatological Science, vol. 43, no. 3, pp. 159-169, 2006.

[4] M. Boguniewicz, "Atopic dermatitis: beyond the itch that rashes," Immunology and Allergy Clinics of North America, vol. 25, no. 2, pp. 333-351, 2005.

[5] K. Kawamoto and H. Matsuda, "Spontaneous mouse model of atopic dermatitis in NC/Nga mice," in Animal Models of Human Inflammatory Skin Diseases, L. S. Chan and, Eds., chapter 25, CRC Press, New York, NY, USA, 2004.

[6] H. Jin, R. He, M. Oyoshi, and R. S. Geha, "Animal models of atopic dermatitis," The Journal of Investigative Dermatology, vol. 129, no. 1, pp. 31-40, 2009. 
[7] H. Matsuda, N. Watanabe, G. P. Geba et al., "Development of atopic dermatitis-like skin lesion with IgE hyperproduction in NC/Nga mice," International Immunology, vol. 9, no. 3, pp. 461-466, 1997.

[8] M. Matsumoto, C. Ra, K. Kawamoto et al., "IgE hyperproduction through enhanced tyrosine phosphorylation of janus kinase 3 in NC/Nga mice, a model for human atopic dermatitis," Journal of Immunology, vol. 162, no. 2, pp. 10561063, 1999.

[9] M. Matsumoto, A. Itakura, A. Tanaka, C. Fujisawa, and H. Matsuda, "Inability of IL-12 to down-regulate IgE synthesis due to defective production of IFN- $\gamma$ in atopic NC/Nga mice," Journal of Immunology, vol. 167, no. 10, pp. 5955-5962, 2001.

[10] C. Vestergaard, H. Yoneyama, M. Murai et al., "Overproduction of Th2-specific chemokines in NC/Nga mice exhibiting atopic dermatitis-like lesions," Journal of Clinical Investigation, vol. 104, no. 8, pp. 1097-1105, 1999.

[11] A. Aioi, H. Tonogaito, H. Suto et al., "Impairment of skin barrier function in NC/Nga Tnd mice as a possible model for atopic dermatitis," British Journal of Dermatology, vol. 144, no. 1, pp. 12-18, 2001.

[12] M. Tominaga, H. Ogawa, and K. Takamori, "Decreased production of semaphorin $3 \mathrm{~A}$ in the lesional skin of atopic dermatitis," British Journal of Dermatology, vol. 158, no. 4, pp. 842-844, 2008.

[13] J. Yamaguchi, F. Nakamura, M. Aihara et al., "Semaphorin3A alleviates skin lesions and scratching behavior in NC/Nga mice, an atopic dermatitis model," Journal of Investigative Dermatology, vol. 128, no. 12, pp. 2842-2849, 2008.

[14] K. Jung, A. Tanaka, H. Fujita et al., "PPAR $\gamma$-mediated suppression of dendritic cells function prevents the onset of $\mathrm{t}$ atopic dertmatitis in NC/NgaTnd mice," Journal of Allergy and Clinical Immunology. In press.

[15] T. Takada, H. Shitara, K. Matsuoka et al., "A novel hairless mouse model on an atopic dermatitis-prone genetic background generated by receptor-mediated transgenesis," Transgenic Research, vol. 17, no. 6, pp. 1155-1162, 2008.

[16] I. Ishii, S. Kurozumi, K. Orito, and H. Matsuda, "Automatic scratching pattern detection for laboratory mice using highspeed video images," IEEE Transactions on Automation Science and Engineering, vol. 5, no. 1, pp. 176-182, 2008.

[17] Y. Nie, I. Ishii, K. Yamamoto, K. Orito, and H. Matsuda, "Realtime scratching behavior quantification system for laboratory mice using high-speed vision," Journal of Real-Time Image Processing, vol. 4, no. 2, pp. 181-190, 2009.

[18] A. Tanaka, S. Muto, K. Jung, A. Itai, and H. Matsuda, "Topical application with a new NF- $\kappa \mathrm{B}$ inhibitor improves atopic dermatitis in NC/NgaTnd mice," Journal of Investigative Dermatology, vol. 127, no. 4, pp. 855-863, 2007.

[19] H. Tanizaki, N. Kambe, Y. Nakamura, A. Tanaka, H. Matsuda, and Y. Miyachi, "Oral administration of bepotastine besilate suppressed scratching behavior of atopic dermatitis model $\mathrm{NC/Nga} \mathrm{mice,"} \mathrm{International} \mathrm{Archives} \mathrm{of} \mathrm{Allergy} \mathrm{and} \mathrm{Immunol-}$ ogy, vol. 145, no. 4, pp. 277-282, 2008.

[20] A. Tanaka, Y. Fukushima, J. Benyacoub, S. Blum, and H. Matsuda, "Prophylactic effect of oral administration of Lactobacillus johnsonii NCC533 (La1) during the weaning period on atopic dermatitis in NC/NgaTnd mice," European Journal of Dermatology, vol. 18, no. 2, pp. 136-140, 2008.

[21] A. Tanaka, K. Jung, J. Benyacoub et al., "Oral supplementation with Lactobacillus rhamnosus CGMCC 1.3724 prevents development of atopic dermatitis in NC/NgaTnd mice possibly by modulating local production of IFN- $\gamma$," Experimental Dermatology, vol. 18, no. 12, pp. 1022-1027, 2009. 


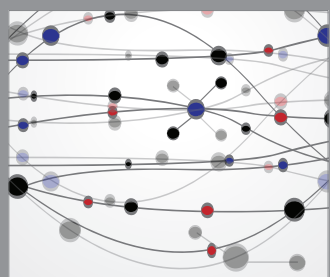

The Scientific World Journal
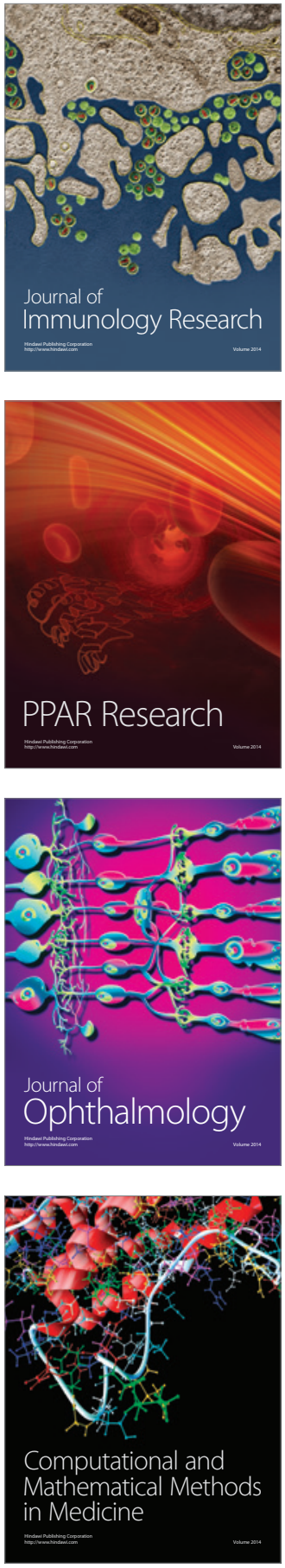

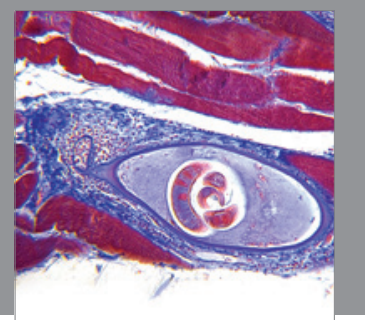

Gastroenterology

Research and Practice
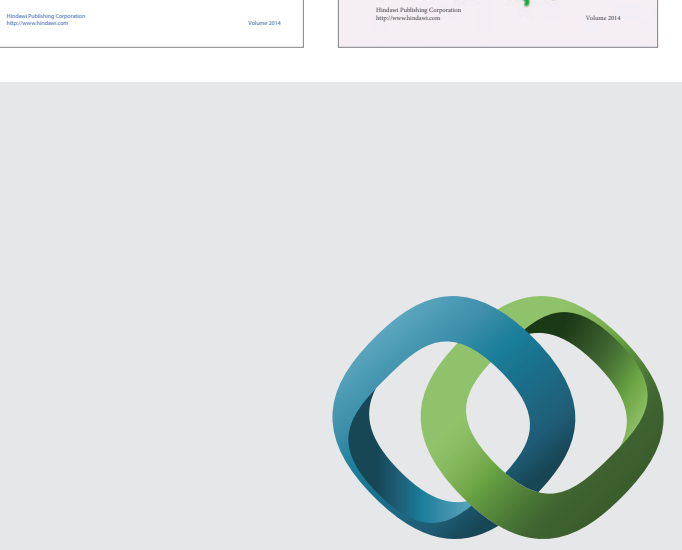

\section{Hindawi}

Submit your manuscripts at

http://www.hindawi.com
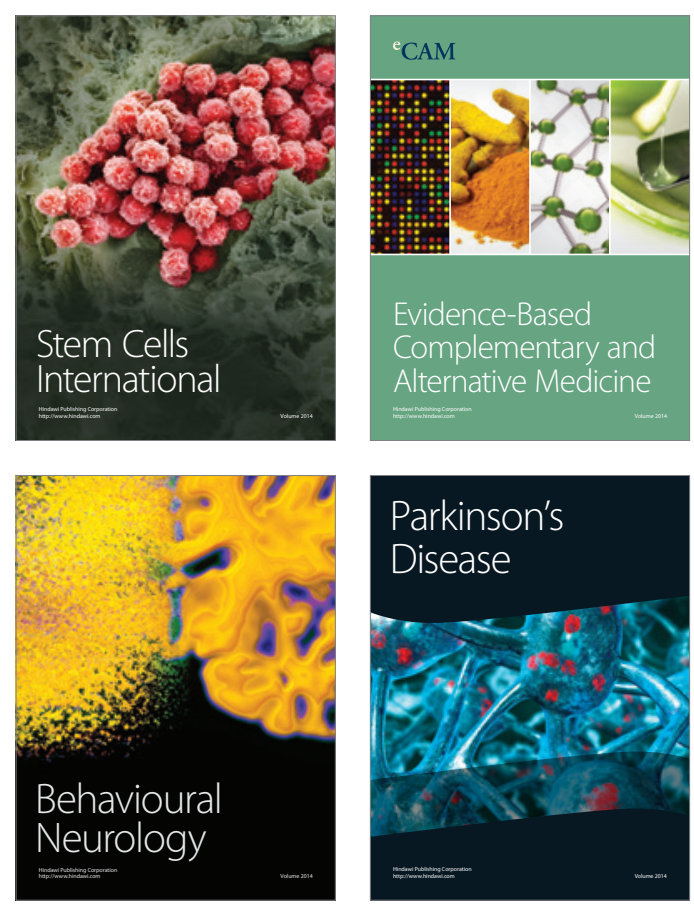

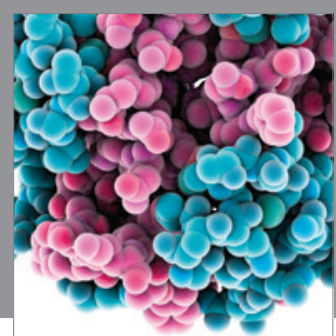

Journal of
Diabetes Research

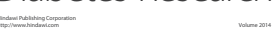

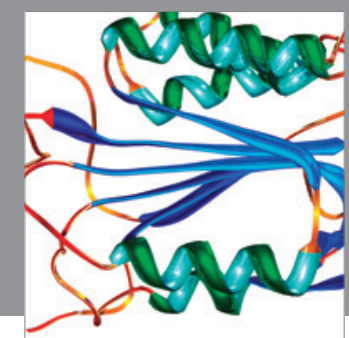

Disease Markers
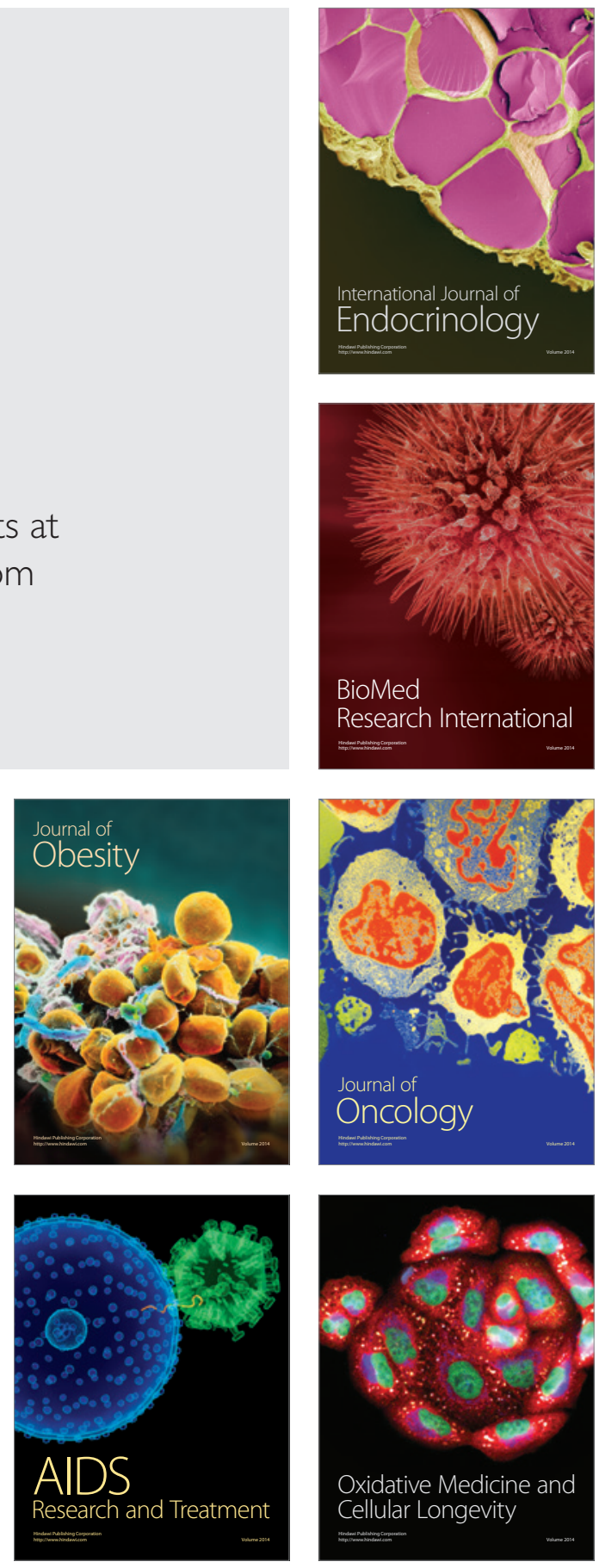